\title{
Incidence and costs of hospitalized adult influenza patients in The Netherlands: a retrospective observational study
}

\author{
Sierk D. Marbus ${ }^{1}$. Valentijn A. Schweitzer ${ }^{2}$. Geert H. Groeneveld ${ }^{3}$. Jan J. Oosterheert ${ }^{4} \cdot$ Peter M. Schneeberger $^{5}$. \\ Wim van der Hoek ${ }^{1}$. Jaap T. van Dissel ${ }^{1,3}$. Arianne B. van Gageldonk-Lafeber ${ }^{1}$. Marie-Josée Mangen ${ }^{1}$
}

Received: 10 May 2019 / Accepted: 25 February 2020 / Published online: 16 March 2020

(c) Springer-Verlag GmbH Germany, part of Springer Nature 2020

\begin{abstract}
Objective Influenza virus infections cause a high disease and economic burden during seasonal epidemics. However, there is still a need for reliable disease burden estimates to provide a more detailed picture of the impact of influenza. Therefore, the objectives of this study is to estimate the incidence of hospitalisation for influenza virus infection and associated hospitalisation costs in adult patients in the Netherlands during two consecutive influenza seasons.

Methods We conducted a retrospective study in adult patients with a laboratory confirmed influenza virus infection in three Dutch hospitals during respiratory seasons 2014-2015 and 2015-2016. Incidence was calculated as the weekly number of hospitalised influenza patients divided by the total population in the catchment populations of the three hospitals. Arithmetic mean hospitalisation costs per patient were estimated and included costs for emergency department consultation, diagnostics, general ward and/or intensive care unit admission, isolation, antibiotic and/or antiviral treatment. These hospitalisation costs were extrapolated to national level and expressed in 2017 euros.

Results The study population consisted of 380 hospitalised adult influenza patients. The seasonal cumulative incidence was 3.5 cases per 10,000 persons in respiratory season 2014-2015, compared to 1.8 cases per 10,000 persons in 2015-2016. The arithmetic mean hospitalisation cost per influenza patient was €6128 (95\% CI €4934-€7737) per patient in 2014-2015 and $€ 8280$ (95\% CI $€ 6254-€ 10,665)$ in 2015-2016, potentially reaching total hospitalisation costs of $€ 28$ million in 2014-2015 and $€ 20$ million in 2015-2016.

Conclusions Influenza virus infections lead to 1.8-3.5 hospitalised patients per 10,000 persons, with mean hospitalisation costs of $€ 6100-€ 8300$ per adult patient, resulting in 20-28 million euros annually in The Netherlands. The highest arithmetic mean hospitalisation costs per patient were found in the 45-64 year age group. These influenza burden estimates could be used for future influenza cost-effectiveness and impact studies.
\end{abstract}

Keywords Influenza $\cdot$ Hospitalisation $\cdot$ Costs $\cdot$ Incidence

JEL Classification I10 $\cdot$ I11

\section{Introduction}

Influenza virus infections cause a high disease and economic burden during seasonal epidemics. The World Health Organization (WHO) estimated that seasonal influenza epidemics

Electronic supplementary material The online version of this article (https://doi.org/10.1007/s10198-020-01172-1) contains supplementary material, which is available to authorized users.

Sierk D. Marbus

sierk.marbus@rivm.nl

Extended author information available on the last page of the article caused annually 3-5 Mio. cases of severe disease [1]. Estimates of global seasonal influenza-associated respiratory deaths amount to $4.0-8.8$ per 100,000 persons annually, but wide variation between countries exists [2]. The highest burden of disease due to influenza is seen in the specific high-risk groups: children aged 0-4 years and elderly with underlying comorbidities [3].

In Europe, influenza is the infectious disease with the highest estimated annual burden, responsible for $30 \%$ of the total burden caused by 31 selected infectious diseases [4]. In The Netherlands, the burden was estimated at 16,316 disability-adjusted life years (DALY) in 2016, using an 
incidence- and pathogen-based approach and corrected for underreporting and under-ascertainment [5]. To provide a more detailed picture of the impact of influenza, reliable influenza incidence and cost estimates are necessary. This could help making informed decisions about allocating resources, such as vaccines and antivirals, and planning influenza intervention strategies to limit the spread of influenza and minimize the costs [6].

However, data on direct medical costs and incidence of hospitalized influenza patients are limited. In the United States (US), the average direct medical costs of seasonal influenza epidemics, based on 2003 US population demographics, were estimated at 10.4 billion dollar annually [7]. Dutch cost estimates are only available for total community-acquired pneumonia (CAP) hospitalized patients, but not further specified for influenza virus infections [8-12]. Moreover, incidence for influenza-like illness (ILI) is limited to patients visiting their general practitioner in primary care [13]. Since, reliable influenza incidence calculations and influenza-associated economic costs in secondary care are unavailable in The Netherlands, we estimated incidence of hospitalization for influenza virus infections and associated hospitalization costs for the first time.

\section{Methods}

\section{Study population and period}

The study population consisted of adult patients with a laboratory-confirmed influenza virus infection admitted to three Dutch hospitals [Jeroen Bosch Hospital (JBZ), Leiden University Medical Center (LUMC), and University Medical Center Utrecht (UMCU)] during the two respiratory seasons 2014-2015 and 2015-2016. The respiratory season was defined as the period from week 40 through week 20 the following year. Influenza A(H3N2) was the dominating influenza virus in season 2014-2015, while influenza A(H1N1) pdm09 was dominant in season 2015-2016 [14, 15]. JBZ is a large regional hospital with 575 beds in the south-eastern part of The Netherlands. LUMC (585 beds) and UMCU ( \pm 1100 beds) are large tertiary academic hospitals, situated in the western part (LUMC) and central part (UMCU) of The Netherlands. The inclusion criteria for patients were: (1) hospital admission for severe acute respiratory infection (SARI) according to the WHO SARI case definition [16], (2) being 18 years or older, and (3) having a positive influenza virus test using real-time polymerase chain reaction (RT-PCR) [17]. Taking into account the Dutch Working Party on Antibiotic Policy (SWAB) guidelines [18] (JBZ and UMCU) and local hospital guidelines (LUMC), all influenza virus tests were requested at the discretion of the treating physician depending on the differential diagnosis. It has to be noted that the SWAB guidelines on influenza primarily focus on the preferred swabbing location, antiviral treatment, and antibiotic treatment of $S$. aureus in ICU patients during the influenza season. Patients hospitalized shorter than $24 \mathrm{~h}$, readmissions within ten days, and patients with hospitalacquired pneumonia were excluded. In case of a readmission within ten days, the initial admission was included and only the re-admission excluded. In The Netherlands, an influenza epidemic is declared if the incidence of medically attended ILI in primary care is above the threshold of 5.1 per 10,000 persons per week for at least two consecutive weeks in combination with the detection of influenza virus in combined nasal and throat swabs from a selection of ILI patients [19].

\section{Data collection}

Data were collected retrospectively from electronic patient records in all three hospitals. The dataset consisted of age, gender, comorbidities (for details see supplemental file Table S1), influenza virus type (A/B), length of hospital stay (LOS) in days, intensive care unit (ICU) admission (yes/no, and length of ICU stay in days), droplet and contact isolation (yes/no), antibiotic therapy (yes/no), oseltamivir treatment (yes/no), and in-hospital mortality (yes/no, date) (Table 1). Patients were stratified by age into four categories (18-44, $45-64,65-74$ and $\geq 75$ years) and by risk of severe or complicated influenza illness into three groups (low, medium and high risk), based on the presence of comorbidities [10, 20, 21] (supplemental file Table S1). It was not feasible to collect data on influenza vaccination status, because it is not routinely documented in electronic patient records and a national influenza vaccination registry is not in place in The Netherlands.

\section{Incidence estimate}

Incidence per respiratory season was calculated as the number of influenza patients admitted to the hospital per week, divided by the catchment population of the three hospitals and expressed per 10,000 persons. The catchment population was calculated by dividing the total number of hospitalizations due to respiratory tract infection (RTI) in each hospital by the total hospitalizations because of RTI in The Netherlands and multiplying this proportion by the total Dutch population size for each year available [22]. It is important to note that it concerns the catchment population for RTI only, and is, therefore, not applicable for incidence calculations related to other type of disease [6]. The data required for the calculation of the catchment population of each hospital were provided by the National Register of hospital discharge diagnosis (Dutch Hospital Data) (supplemental file Table S2). A selection of International Statistical Classification of Diseases and Related Health Problems (ICD-10) 
Table 1 Baseline characteristics of influenza patients during respiratory seasons 2014-2015 and 2015-2016

\begin{tabular}{|c|c|c|c|c|}
\hline & General hospital & Academic hospitals & $p$ value* & Total \\
\hline Admission, $n$ & 102 & 278 & & 380 \\
\hline Age, median (IQR) & $75(59-84)$ & $62(48-74)$ & 0.00 & $64.5(52-77)$ \\
\hline \multicolumn{5}{|l|}{ Age category $n(\%)$ (years) } \\
\hline $18-44$ & $7(6.9)$ & $56(20.1)$ & 0.00 & $63(16.6)$ \\
\hline $45-64$ & $30(29.4)$ & $98(35.3)$ & & $128(33.7)$ \\
\hline $65-74$ & $16(15.7)$ & $55(19.8)$ & & $71(18.7)$ \\
\hline$\geq 75$ & $49(48.0)$ & $69(24.8)$ & & $118(31.1)$ \\
\hline Male, $n(\%)$ & $48(47.1)$ & $170(61.2)$ & 0.01 & $218(57.4)$ \\
\hline Female, $n(\%)$ & $54(52.9)$ & $108(38.8)$ & & $162(42.6)$ \\
\hline \multicolumn{5}{|l|}{ Risk group, $n(\%)$} \\
\hline Low & $17(16.7)$ & $31(11.1)$ & 0.00 & $48(12.6)$ \\
\hline Medium & $58(56.9)$ & $66(23.7)$ & & $124(32.6)$ \\
\hline High & $27(26.5)$ & $181(65.1)$ & & $208(54.7)$ \\
\hline Number of comorbidities, median $n(\mathrm{IQR})^{\mathrm{a}}$ & $1(1-2)$ & $1(1-3)$ & 0.53 & $2(1-2)$ \\
\hline ICU, $n(\%)$ & $20(19.6)$ & $50(18.0)$ & 0.72 & $70(18.4)$ \\
\hline Length of hospital stay (LOS), median (IQR) & $9(5-15)$ & $4(2-8)$ & 0.00 & $5(3-11)$ \\
\hline General ward days per patient & $8(5-13)$ & $3(2-6)$ & 0.00 & $5(3-11)$ \\
\hline ICU days per ICU patient & $5(2-11)$ & $5(2-11)$ & 0.97 & $5(2-10)$ \\
\hline \multicolumn{5}{|l|}{ Influenza virus, $n(\%)$} \\
\hline Type A & $80(78.4)$ & $214(77.0)$ & 0.15 & $294(77.4)$ \\
\hline Type B & $22(21.6)$ & $54(19.4)$ & & $76(20.0)$ \\
\hline Type A and B & $0(0)$ & $10(3.6)$ & & $10(2.6)$ \\
\hline Antibiotic treatment, $n(\%)^{\mathrm{b}}$ & $73(73.7)$ & $224(80.6)$ & 0.15 & $297(78.8)$ \\
\hline Oseltamivir treatment, $n(\%)^{\mathrm{c}}$ & $46(46.5)$ & $142(51.1)$ & 0.43 & $188(49.9)$ \\
\hline In-hospital mortality, $n(\%)$ & $5(4.9)$ & $16(5.8)$ & 0.75 & $21(5.5)$ \\
\hline
\end{tabular}

*Statistical difference tested between general hospital and academic hospitals using unpaired $t$ test, Mann-Whitney $U$ test, Kruskal-Wallis test or Chi-square test

${ }^{a}$ Missing general hospital: $n=0$, academic hospital: $n=118$

${ }^{\mathrm{b}}$ Missing general hospital: $n=3$, academic hospital: $n=0$

${ }^{\mathrm{c}}$ Missing general hospital: $n=3$, academic hospital: $n=0$

codes related to RTI (J00-J22, A15, A16, A48.1, A70 and A78) was determined for each hospital for the years 2014, 2015 and 2016 (supplemental file Figure S1 footnote) [22, 23]. Taking into account the non-normal distribution of the catchment population over the available respiratory seasons, we used the median value for our incidence calculations.

\section{Costs estimates}

Hospitalization costs were estimated using a bottom-up approach and included costs for the emergency department (ED) visit, requested diagnostics during stay on the ED, hospitalization stay in a general ward and the ICU, medication costs for antibiotic and oseltamivir treatment, isolation in general ward rooms and associated costs. Associated isolation costs involve the use of gloves, Free Flight Phase 1 (FFP1) masks, and additional work load for medical and cleaning personnel and were not included in the daily costs of admission on a regular ward or ICU.

Hospitalization costs were calculated by multiplying recorded units of used healthcare resources with corresponding unit prices (supplemental file Table S3). Unit prices for laboratory tests were retrieved form the Dutch Healthcare Authority [24], medication prices from the National Health Care Institute [25], and medical consultations and hospital admissions were estimated using Dutch reference prices [26], using the weighted mean unit cost for hospital admission. The weighted mean unit cost takes into account the distribution of general ward and academic hospital beds in The Netherlands. Diagnostics for patients with an RTI on an emergency ward were assumed to include a chest X-ray and laboratory diagnostic tests, including full blood examination, respiratory viral PCR panel, and urine analysis (see supplemental file, Table S3). The urine analysis included a urine screening test and a pneumococcal urinary antigen 
test. For ICU patients, diagnostics, isolation and medication costs were already included in the total ICU admission costs. Costs for antibiotic treatment on the general ward were calculated for average antibiotic therapy course of one week in line with the most recent SWAB guidelines [18]. Different antibiotic SWAB treatment regimens on the general ward for mild and moderate severe CAP ( $\beta$-lactam antibiotic) versus severe CAP ( $2 \mathrm{nd} / 3 \mathrm{rd}$ generation cephalosporin) were chosen for costs estimations of antibiotic treatment [27]. Medication costs for oseltamivir on the general ward were calculated for the duration of 5 days according to SWAB guidelines. A bottom-up calculation was done for the additional costs for isolation in the first week of admission in the general ward, including residing in single rooms, additional associated workload for medical personnel, and usage of FFP1 masks [28, 29].

Arithmetic mean hospitalization costs per adult influenza patient were estimated and extrapolated to the national level. We attempted to obtain annual national hospitalization costs attributable to influenza by multiplying the estimated influenza incidence with the estimated average hospital costs per influenza patient. We could not exclude that the number of more severely affected patients, resulting in an extremely prolonged hospital stay, might have been overrepresented in our study sample. Therefore, geometric mean hospitalization costs were calculated and used as a sensitivity analysis to take into account skewed data with several outliers (supplemental file Table S4). Costs were presented in 2017 euros and, if necessary, updated using the Dutch Consumer Price index [30].

We performed a sensitivity analysis to examine to what extent the exclusion of influenza patients admitted shorter than $24 \mathrm{~h}$ influenced our cost analysis. For the sensitivity analysis, we collected additional data on influenza patients admitted shorter than $24 \mathrm{~h}$ in one hospital (LUMC). The proportion of patients admitted shorter than $24 \mathrm{~h}$ versus admitted longer than $24 \mathrm{~h}$ in LUMC was used as a proxy for the other two hospitals (JBH and UMCU). Arithmetic mean hospitalization costs per influenza patient was calculated per subgroup (admitted $<24 \mathrm{~h}$, admitted $>24 \mathrm{~h}$ ) (supplemental file Table S5).

In addition, to provide more insight into the differences of hospitalization costs between risk groups, we reviewed electronic patients' records for detailed information on complications in a proportion of patients (21\%) with the top highest hospitalization costs.

\section{Statistics}

Descriptive statistics were used for reporting the baseline characteristics of the study population, such as total number, percentage per category, median and interquartile range (IQR). Arithmetic and geometric means were used for hospitalization cost data. A Chi-square test was used to compare categorical variables. A Mann-Whitney $U$ test, Kruskal-Wallis test, or unpaired $T$ test was performed for numerical variables depending on their distribution and number of groups. Missing values were imputed using multiple imputation, creating five imputed datasets. Bootstrapping with 1000 iterations was used to determine 95\% confidence intervals (CI). A $p$ value of $<0.05$ was considered significant. Data were analyzed using IBM SPSS Statistics, Version 24.

\section{Results}

\section{Study population}

A total number of 380 influenza patients were admitted to the three Dutch hospitals (catchment population 718,000 persons) during respiratory seasons 2014-2015 and 2015-2016 (Table 1). The median number of all-cause hospitalizations was approximately 31,000 in the three hospitals in the period 2014-2016. The study patients had a median age of 65 years and most patients belonged to the $45-64$ and $\geq 75$ age groups (respectively, $34 \%$ and $31 \%$ ). The influenza patients had a median of two comorbidities and were classified into the medium- and high-risk groups in, respectively, $33 \%$ and $55 \%$ of the cases. The most prevalent comorbidities were cardiovascular disease (50\%), followed by immunodeficiency due to disease or medication $(35 \%)$ and chronic respiratory disease $(35 \%)$. The median length of hospital stay was 5 days and $18 \%$ of the patients were admitted to ICU. In both respiratory seasons, influenza A virus was the most common influenza type. A high proportion of influenza patients were treated with antibiotics (79\%), because of a suspected bacterial superinfection as a complication of influenza. The total in-hospital mortality for influenza patients was $6 \%$ during two consecutive respiratory seasons (supplemental file Table S6).

\section{Incidence}

In respiratory season 2014-2015, the seasonal cumulative incidence was 3.5 cases per 10,000 persons and the incidence peaked at 0.3 cases per 10,000 persons in week 5 . The seasonal cumulative incidence was 1.8 cases per 10,000 persons in respiratory season 2015-2016 and the incidence for hospitalized influenza patients ( 0.3 cases per 10,000 persons) peaked at week 7 (supplemental file Figure S1).

\section{Hospitalization costs per patient}

The arithmetic mean hospitalization costs per influenza patient was €6128 (95\% CI €4934-€7737) in 2014-2015, 
Fig. 1 Arithmetic mean hospitalization costs per influenza patient admitted to three Dutch hospitals during respiratory seasons 2014-2015 and 2015-2016. Error bars indicate the $95 \%$ confidence interval as determined by bootstrapping

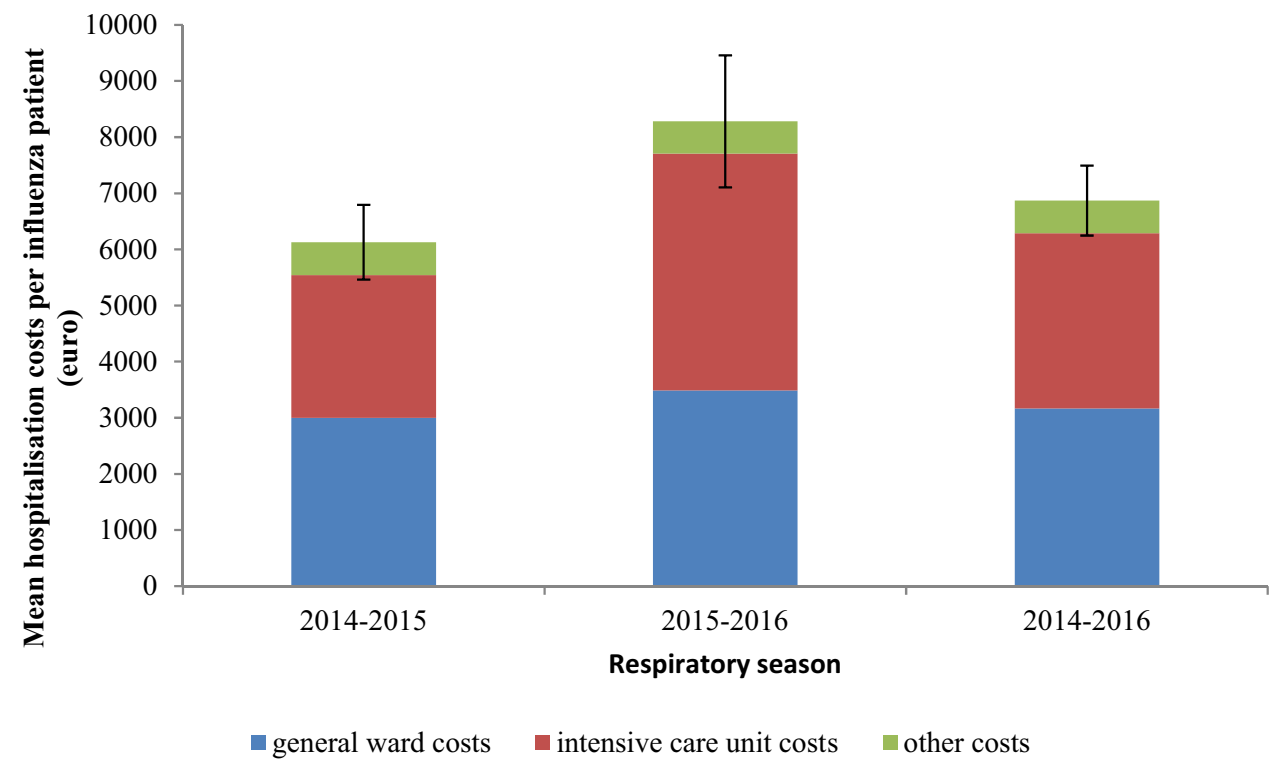

Table 2 Arithmetic mean hospitalization costs per hospital type (general and academic) per influenza patient during respiratory seasons 2014-2015 and 2015-2016

\begin{tabular}{llll}
\hline Hospital & Patients $(N)$ & $\begin{array}{l}\text { Hospitalization costs per patient } \\
(2017 \text { euros) }\end{array}$ \\
\cline { 3 - 4 } & & Arithmetic mean & $95 \%$ CI \\
\hline General hospital $^{\mathrm{a}}$ & 102 & 8247 & $6583-10,206$ \\
Academic hospital $^{\mathrm{b}}$ & 278 & 6365 & $5078-8132$ \\
Total & 380 & 6870 & $5779-7994$ \\
\hline
\end{tabular}

* Statistically significant difference between general hospital and academic hospitals using a Mann-Whitney $U$ test; $p=0.00$

a JBZ

${ }^{\mathrm{b}} \mathrm{LUMC}$ and UMCU
$€ 8280$ (95\% CI €6254-€10,665) in 2015-2016 and €6870 (95\% CI €5750-€8071) in 2014-2016 (Fig. 1). No significant differences in hospitalization costs between the two seasons were found ( $p=0.58$ ) (supplemental file Table S7). General ward and ICU costs accounted for, respectively, $46 \%$ and $45 \%$ of the mean hospitalization costs per patient. In both respiratory seasons, the arithmetic mean hospitalization costs per patient in the general hospital $(€ 8247,95 \%$ CI $€ 6583-€ 10,206)$ were significantly $(p=0.00)$ higher than in the university hospitals ( $€ 6365,95 \%$ CI $€ 5078-€ 8132)$ (Table 2).

The arithmetic mean hospitalization costs were highest for the 45-64-year age group (€8970, 95\% CI $€ 6610-€ 11,694$ ) (Fig. 2, supplemental file Table S8) and
Fig. 2 Arithmetic mean hospitalization costs per age category per influenza patient admitted to three Dutch hospitals during respiratory seasons 2014-2015 and 2015-2016. Error bars indicate the $95 \%$ confidence interval as determined by bootstrapping

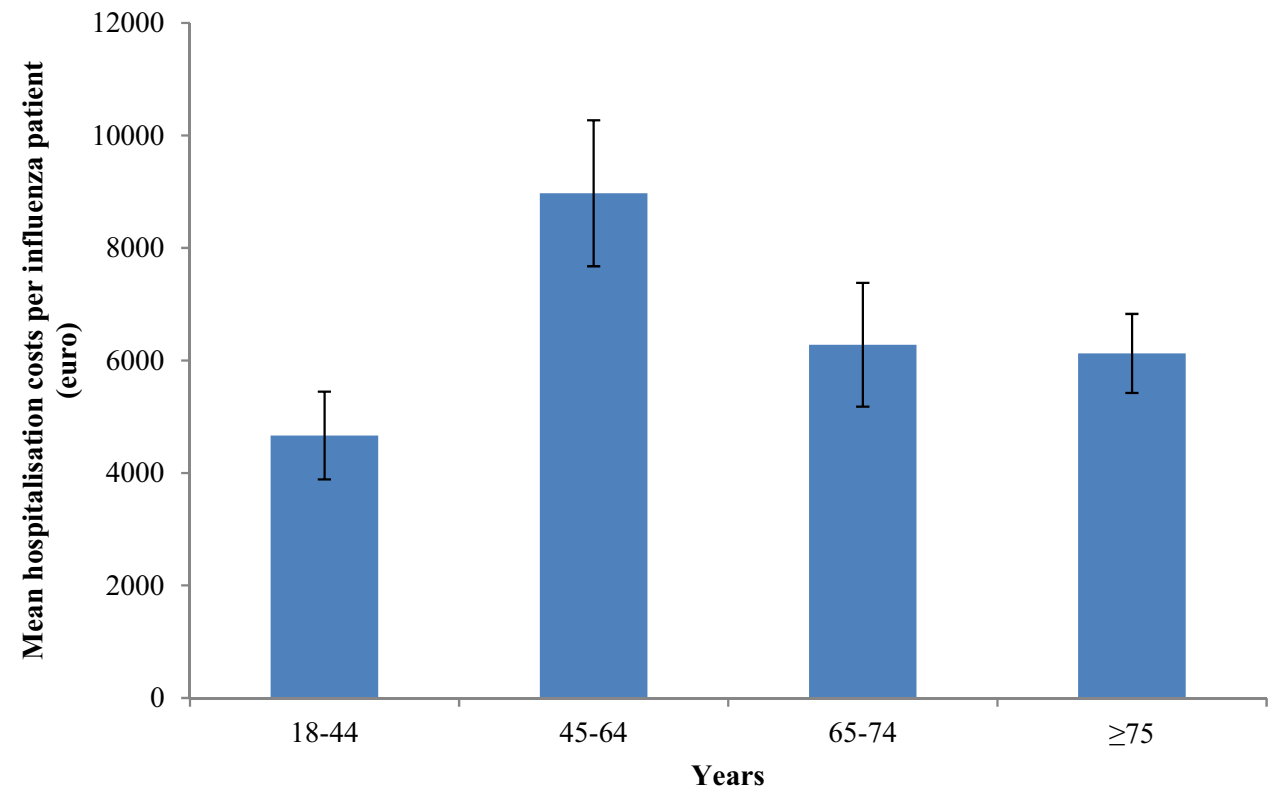


the low-risk group ( $€ 14,249,95 \%$ CI $€ 8798-€ 21,345)$ during respiratory seasons 2014-2015 and 2015-2016 (Table 3). The 45-64-year age group had the highest percentage of ICU admissions (8.4\%) and the longest median ICU stay per admitted ICU patient ( 8 days). The low-risk group had the longest median LOS per patient (7 days) and longest median stay in ICU (18 days) (supplemental file Table S9). Analysis within the low-risk group with high hospitalization costs reported an outlier $(€ 134,395)$ in 2014-2015, which belonged to a 53-year-old ICU patient with the longest LOS (86 days; including both ward and ICU days). If this patient was excluded from the analysis, the arithmetic mean hospitalization costs per influenza patient amounted to €6534 (95\% CI €5570-€7510) in 2014-2016.

Acute respiratory distress syndrome (ARDS), as a complication of an influenza virus infection, was diagnosed more frequently in the low-risk group (26\%) versus the medium (12\%)- and high-risk group (1\%). In comparison to influenza patients with other complications (e.g., sepsis, acute renal injury requiring dialysis, heart failure), ARDS patients also had the longest median LOS (29 days).

If geometric mean hospitalization costs were used, the total hospitalization costs per influenza patient were $€ 3601$ (95\% CI €3217-€4030) in 2014-2015, €4099 (95\% CI $€ 3398-€ 4944)$ in 2015-2016, resulting in €3765 (95\% CI $€ 3414-€ 4151$ ) in 2014-2016 (supplemental file Table S4 and S10).

Taking into account influenza patients admitted less than $24 \mathrm{~h}$ and over $24 \mathrm{~h}$, the arithmetic mean hospitalization costs for influenza patients amounted to $€ 6208$ in 2014-2016. If compared to hospitalization cost excluding patients admitted less than $24 \mathrm{~h}(€ 6870)$, an arithmetic mean difference of $€ 662$ per influenza patient was found (supplemental file Table S5).

Table 3 Arithmetic mean hospitalization costs per risk group per influenza patient admitted to three Dutch hospitals during influenza seasons 2014-2016

\begin{tabular}{lccl}
\hline Risk group $^{\mathrm{a}}$ & Total $(N)$ & $\begin{array}{l}\text { Arithmetic mean } \\
\text { costs }(2017 \text { euros)* }\end{array}$ & 95\% CI \\
\hline Low & 48 & 14,249 & $8798-21,345$ \\
Medium & 124 & 6973 & $5453-9206$ \\
High & 208 & 5132 & $4230-6246$ \\
Total & 380 & 6870 & $5819-7983$ \\
\hline
\end{tabular}

* Statistically significant difference between risk groups using a Kruskal-Wallis test; $p=0.01$

${ }^{\text {a }}$ Risk group classification of severe or complicated influenza illness in three groups (low, medium and high risk), based on the presence of comorbidities (supplemental file, Table S1)

\section{Hospitalization costs on national level}

Total hospitalization costs on national level were approximated at $€ 28$ Mio. in 2014-2015 and €20 Mio. in 2015-2016. Using the geometric mean, rather than the arithmetic mean, these costs would approximate to $€ 17$ Mio. in 2014-2015 and €10 Mio. in 2015-2016 (supplemental file Table S4).

\section{Discussion}

\section{Principal results}

To our knowledge, this is the first Dutch study reporting influenza burden estimates in secondary care with regard to incidence and hospitalization costs. Based on seasonal cumulative and peak incidence from three Dutch hospitals, the season 2014-2015 was considered more severe than the season 2015-2016. The arithmetic mean hospitalization costs per influenza patient were $€ 6870$ (95\% CI $€ 5750-€ 8071)$ during respiratory seasons $2014-2016$. If extrapolated to national level, the hospitalization costs of influenza patients amounted to 28 Mio. in 2014-2015 and 20 Mio. in 2015-2016. In addition, the general hospital had significantly higher arithmetic mean hospitalization costs than the two academic hospitals. The highest arithmetic mean hospitalization costs per patient were found in the 45-64year age group and in the low-risk group.

\section{Comparisons with other studies}

\section{Incidence}

Incidence of hospitalization for laboratory-confirmed influenza virus infections in Europe is rarely available [31] and hospitalizations are mostly reported as absolute numbers without denominator [32]. The US Centers for Disease Control and Prevention (CDC) reported an incidence for laboratory-confirmed influenza hospitalizations of 6.4 cases per 10,000 persons in season 2014-2015 and 3.2 per 10,000 in season 2015-2016 [33]. The incidence estimates are considerably higher than reported in our study, which could be due to different available healthcare system in both countries. In the Dutch healthcare system, the general practitioners (GPs) have a gatekeeping role for specialized care. This is illustrated by a Dutch study showing that the vast majority of CAP patients are managed successfully at GP level without hospitalization [34]. In contrast, in the US, approximately two-thirds of the adult hospitalized patients 
with CAP were in a low-risk group with respect to the risk of death, suggesting a low threshold for admitting CAP patients to hospital [35, 36].

\section{Costs}

Comparison with other studies is difficult due to differences in healthcare system, applied resource unit cost, case definition, study population, and/or taking readmissions into account.

Differences in healthcare system and resource unit prices [37-40] are illustrated by a Spanish [41], Italian [42], German [43] and Canadian study [44]. In Spain, despite a longer arithmetic mean LOS (11.1 days), a lower arithmetic mean costs per influenza hospitalization (in 2015 euros) in patients aged $\geq 65$ years $(€ 3219)$ was reported. Italy reported similar mean hospitalization costs for ILI/lower respiratory tract infection in patients 50 years and older (€3353). In Germany, mean influenza hospitalization costs ( $€ 5832$, in 2013 euros) were lower than in our study. In Canada, higher hospitalization costs per influenza patient (in 2015 euros) were found (€10,312, 95\% CI $€ 9776-€ 10,848)$. The longer arithmetic mean LOS (10.8 days) and higher in-hospital mortality (9.3\%), taking into account costs for readmissions within 30 days, may also account for the difference with our study.

Differences in case definition and study population are illustrated by Dutch studies, which focused mainly on community-acquired pneumonia (CAP) $[9,10,45]$ and invasive pneumococcal disease [10] in, respectively, an adult [9, 45] and elderly population [10]. Vissink et al. [10] reported that mean hospitalization costs of an elderly CAP patient amounted to $€ 8081$. In comparison, we found arithmetic mean influenza-related hospitalization costs of $€ 6183$ (95\% CI €4937-€7625) in the same age group. The higher hospitalization costs found by Vissink et al. could be explained by their inclusion of more detailed cost data on diagnostics and treatment during admission and having an older elderly study population (56\% versus $31 \%$ in our study). An additional explanation is that CAP has a great variation of causative pathogens that may result in a longer hospital stay (12.0 days versus 8.5 days in our study) and higher in-hospital mortality ( $11.1 \%$ versus $5.5 \%$ in our study) with consequently higher hospitalization costs. This was also illustrated by the study of Spoorenberg et al., showing that CAP caused by $S$. pneumoniae and $S$. aureus is associated with higher hospitalization costs mainly due to longer duration of hospital stay [9]. They estimated a mean hospitalization costs per CAP patient of $€ 4098$, which is lower than our hospitalization costs per influenza patient. The difference in mean hospitalization costs could be explained by the exclusion of all immunocompromised patients and a lower percentage of ICU admissions ( $7.5 \%$ versus $18.4 \%$ in our study) in the study by Spoorenberg et al.
The differences in hospitalization costs between the general and academic hospitals found in our study are attributed to a longer LOS in the general hospital (JBZ), mainly caused by longer length of stay on the general ward. Although we do not have a definite explanation for this result, several hypotheses could be made. Firstly, it is possible that pressure on hospital beds during influenza epidemics is higher in academic hospitals, resulting in different policies with respect to supported early discharge between the general and academic hospitals. Secondly, the amount of patient transfers to other hospitals could be higher in academic hospitals than in general hospitals. Thirdly, academic hospitals have a lower threshold for admitting immunocompromised patients, who are, therefore, less ill and require a shorter LOS, leading to lower hospitalization costs. On the other hand, general hospitals more often admit patients without significant comorbidities, but are more severely ill and require a longer LOS, with higher associated hospitalization costs.

Similar to the US study of Young-Xu et al. estimating costs of influenza-attributed hospitalizations [46], we found the highest costs in the age group 45-64 years. Although higher hospitalization costs are largely driven by LOS [44], mean LOS in our study was actually higher in the $\geq 75$ age group. Nevertheless, the highest hospitalization costs per patient in the age group 45-64 years can be partly explained by the highest percentage of ICU admissions and the longest stay per admitted ICU patient. In agreement with this result, Rozenbaum et al. found the highest mean hospitalization costs per ICU patient with CAP $(€ 16.374)$ in age group 50-64 years [8].

The low-risk group had the highest hospitalization costs, which could largely be explained by the longest median LOS per patient and ICU days per ICU patient. The higher proportion of ARDS patients found in the low-risk group, compared to the medium- and high-risk group, is most likely responsible for this result. In addition, the main outlier in hospitalization costs belonged to an influenza patient in the low-risk group and in the age group 45-64 years. This influenza patient without comorbidities was admitted to the ICU with sepsis and acute renal failure, requiring dialysis. In contrast to our results, other studies $[41,42,46]$ stated that underlying medical conditions contributed to an increase in total hospitalization costs for adults and elderly. Only one US study in children found lower costs in high-risk patients, but this difference was not significant [47, 48].

By comparing extrapolated mean hospitalization costs of influenza patients to hospitalization costs of other diseases, our estimations could be put into context. Our estimated total hospitalization costs of influenza on national level (in 2017 euros) is in the same range as gastro-intestinal infections (21 Mio. euros), but lower than for depression ( 30 Mio., including medication costs) in The Netherlands [49]. In contrast to influenza hospitalization costs, it has to be 
noted that total hospitalization costs due to gastro-intestinal infections include multiple causative pathogens.

\section{Strengths and limitations}

A strength of this study is that we could dispose of accurate catchment populations of each hospital. Therefore, for the first time, we could report incidence of hospitalized influenza patients in The Netherlands. Another strength is that we took differences in unit prices for admission to the general ward, in both general as academic hospital settings, into account, using weighted mean for calculation of hospitalization costs. There are also several limitations that have to be taken into account in this study. Our seasonal cumulative incidence for influenza hospitalizations is based on three Dutch hospitals, which might not be representative for the incidence and associated mean hospitalization costs in the entire Dutch population. It is also important to note that the overall arithmetic mean hospitalization costs per influenza patient are likely to be an underestimation of the true costs, caused by unavailable cost indicators. Firstly, we did not take into account patient transfers to other hospitals because of capacity problems during the influenza epidemic or readmissions of the influenza patient. This could have underestimated our mean hospitalization costs to some extent. Secondly, excluding influenza patients admitted less than $24 \mathrm{~h}$, underestimated our incidence estimates and our national hospitalization costs as well; whereas, the mean hospitalization cost per admitted influenza patient was overestimated. Thirdly, 'other costs' were underestimated in our analysis, because insufficient data were collected on treatment and diagnostics during admission. To compensate for this, assumptions had to be made for the duration of isolation, empiric antibiotic therapy and duration, and oseltamivir treatment. Data on additional treatment and use of diagnostics because of complications during hospital admission were not available in our dataset and, therefore, disregarded in our cost estimate. For one severe outlier, we retrieved additional information on complications during hospital stay resulting in better insight in the mean hospitalization costs. Fourthly, no data were available on hospitalized patients with an RTI with negative influenza virus test results. Even with RT-PCR, false-negative test results may occur due to improper or poor sample collection, handling and/or processing [50]. False-negative influenza test results could have underestimated the total hospitalization costs per influenza patient. Finally, this study was purely limited to hospitalization costs. Information on healthcare use before and after hospital admission, on patient costs and related productivity losses were absent and, therefore, not considered in the current study. Extrapolation of mean hospitalization costs of influenza patients to national level is a rough estimation and has to be interpreted with caution, because the representativeness of the study hospitals is unknown and outliers could have resulted in overestimation of the total hospitalization costs.

\section{Conclusions}

Influenza virus infections lead to $1.8-3.5$ hospitalized patients per 10,000 persons, with mean hospitalization costs of $€ 6100-€ 8300$ per adult patient, resulting in 20-28 Mio. euros annually in The Netherlands. The highest arithmetic mean hospitalization costs per patient were found in the 45-64-year age group. These influenza burden estimates could be used for future influenza cost-effectiveness and impact studies.

Acknowledgements We thank Noor Ismail, Grazia Rooth and Claudia de Wilde for their cooperation in the data collection. We thank Anita Suijkerbuijk for critically reading the manuscript.

Author contributions SM designed the study, analyzed and interpreted the data, and drafted the manuscript. VS, GG, J-JO, PS, RVG-L, and WVDH participated in the design and coordination of the study, and editing the manuscript. JVD participated in editing the manuscript. M-JM participated in the design of the study, performing statistical analysis, interpretation and editing of the manuscript. All authors read and approved the manuscript.

Funding None.

Data availability Data can be made available for peer review on reasonable request through contacting the corresponding author.

\section{Compliance with ethical standards}

Conflict of interest The authors declare that they have no competing interests.

Ethics approval The Dutch Medical Research Involving Human Subjects Act did not apply to this study, because anonymous data were used and there were no interventions other than routine clinical care. A waiver for full medical ethical review was obtained from the Medical Ethical Committee UMC Utrecht (reference number WAG/ $\mathrm{mb} / 16 / 019885$ ), LUMC (reference number G16.054/NV/nv) and JBZ (reference number 2016.07.06.01).

\section{References}

1. WHO: Influenza (seasonal) fact sheet. https://www.who.int/newsroom/fact-sheets/detail/influenza-(seasonal) (2018). Accessed 8 Oct 2018

2. Iuliano, A.D., Roguski, K.M., Chang, H.H., Muscatello, D.J., Palekar, R., Tempia, S., Cohen, C., Gran, J.M., Schanzer, D., Cowling, B.J., Wu, P., Kyncl, J., Ang, L.W., Park, M., RedlbergerFritz, M., Yu, H., Espenhain, L., Krishnan, A., Emukule, G., van Asten, L., Pereira da Silva, S., Aungkulanon, S., Buchholz, U., Widdowson, M.A., Bresee, J.S.: Estimates of global seasonal influenza-associated respiratory mortality: a modelling study. 
Lancet 391(10127), 1285-1300 (2018). https://doi.org/10.1016/ s0140-6736(17)33293-2

3. Heymann, D.L.: Control of Communicable Diseases Manual. American Public Health Association, Washington, DC (2015)

4. Cassini, A., Colzani, E., Pini, A., Mangen, M.J., Plass, D., McDonald, S.A., Maringhini, G., van Lier, A., Haagsma, J.A., Havelaar, A.H., Kramarz, P., Kretzschmar, M.E., On Behalf Of The, B.C.: Impact of infectious diseases on population health using incidence-based disability-adjusted life years (DALYs): results from the Burden of Communicable Diseases in Europe study, European Union and European Economic Area countries, 2009 to 2013. Eur. Commun. Dis. Bull. 23, 16 (2018). https://doi. org/10.2807/1560-7917.es.2018.23.16.17-00454

5. RIVM: State of Infectious Diseases in The Netherlands. https:// www.rivm.nl/en/Documents_and_publications/Scientific/Repor ts/2018/september/State_of_infectious_diseases_in_the_Nethe rlands_2017 (2017). Accessed 8 Oct 2018

6. WHO: A Manual for Estimating Disease Burden Associated with Seasonal Influenza. https://www.who.int/influenza/resources/publi cations/manual_burden_of_disease/en/ (2015). Accessed 8 Oct 2018

7. Molinari, N.A., Ortega-Sanchez, I.R., Messonnier, M.L., Thompson, W.W., Wortley, P.M., Weintraub, E., Bridges, C.B.: The annual impact of seasonal influenza in the US: measuring disease burden and costs. Vaccine 25(27), 5086-5096 (2007). https://doi. org/10.1016/j.vaccine.2007.03.046

8. Rozenbaum, M.H., Mangen, M.J., Huijts, S.M., van der Werf, T.S., Postma, M.J.: Incidence, direct costs and duration of hospitalization of patients hospitalized with community acquired pneumonia: a nationwide retrospective claims database analysis. Vaccine 33(28), 3193-3199 (2015). https://doi.org/10.1016/j.vacci ne.2015.05.001

9. Spoorenberg, S.M., Bos, W.J., Heijligenberg, R., Voorn, P.G., Grutters, J.C., Rijkers, G.T., van de Garde, E.M.: Microbial aetiology, outcomes, and costs of hospitalisation for communityacquired pneumonia; an observational analysis. BMC Infect. Dis. 14, 335 (2014). https://doi.org/10.1186/1471-2334-14-335

10. Vissink, C.E., Huijts, S.M., de Wit, G.A., Bonten, M.J., Mangen, M.J.: Hospitalization costs for community-acquired pneumonia in Dutch elderly: an observational study. BMC Infect. Dis. 16, 466 (2016). https://doi.org/10.1186/s12879-016-1783-9

11. Mangen, M.J., Rozenbaum, M.H., Huijts, S.M., van Werkhoven, C.H., Postma, D.F., Atwood, M., van Deursen, A.M., van der Ende, A., Grobbee, D.E., Sanders, E.A., Sato, R., Verheij, T.J., Vissink, C.E., Bonten, M.J., de Wit, G.A.: Cost-effectiveness of adult pneumococcal conjugate vaccination in The Netherlands. Eur Respir J 46(5), 1407-1416 (2015). https://doi. org/10.1183/13993003.00325-2015

12. Mangen, M.J., Rozenbaum, M.H., Huijts, S.M., van Werkhoven, C.H., Postma, D.F., Atwood, M., van Deursen, A.M., van der Ende, A., Grobbee, D.E., Sanders, E.A., Sato, R., Verheij, T.J., Vissink, C.E., Bonten, M.J., de Wit, G.A.: Supplementary material-cost-effectiveness of adult pneumococcal conjugate vaccination in The Netherlands. Eur Respir J 46(5), 1-26 (2015)

13. NIVEL: NIVEL Primary Care Database. https://www.nivel.nl/en/ nivel-primary-care-database (2018). Accessed 8 Oct 2018

14. Teirlink, A.C., van Asten, L., Brandsema, P.S., et al: Annual report surveillance of influenza and other respiratory infections in The Netherlands: winter 2014/2015. In: National Institute for Public Health and the Environment (RIVM), Bilthoven (2015)

15. Teirlink, A.C., van Asten, L., Brandsema, P.S., et al: Annual report surveillance of influenza and other respiratory infections in The Netherlands: winter 2015/2016. In: National Institute for Public Health and the Environment (RIVM), Bilthoven (2016)

16. WHO: WHO Surveillance Case Definitions for ILI and SARI. https://www.who.int/influenza/surveillance_monitoring/ ili_sari_surveillance_case_definition/en/ (2014). Accessed 13 Mar 2018

17. Boivin, G., Cote, S., Dery, P., De Serres, G., Bergeron, M.G.: Multiplex real-time PCR assay for detection of influenza and human respiratory syncytial viruses. J. Clin. Microbiol. 42(1), 45-51 (2004)

18. Stichting-Werkgroep·Antibiotica-Beleid: SWAB-Onderste Luchtwegen. https://www.swab.nl/swab/cms3.nsf/uploads/6A6E1 27F9A2C1168C125816F004A013A/\$FILE/CAP_SWAB_2017DEF_R5.pdf (2018). Accessed 11 June 2018

19. Vega, T., Lozano, J.E., Meerhoff, T., Snacken, R., Beaute, J., Jorgensen, P., Ortiz de Lejarazu, R., Domegan, L., Mossong, J., Nielsen, J., Born, R., Larrauri, A., Brown, C.: Influenza surveillance in Europe: comparing intensity levels calculated using the moving epidemic method. Influenza Other Respir. Viruses 9(5), 234-246 (2015). https://doi.org/10.1111/irv.12330

20. WHO: People at High Risk of Developing Serious Flu-Related Complications. https://www.cdc.gov/flu/about/disease/high_risk. htm (2018). Accessed 10 Oct 2018

21. Mertz, D., Kim, T.H., Johnstone, J., Lam, P.P., Science, M., Kuster, S.P., Fadel, S.A., Tran, D., Fernandez, E., Bhatnagar, N., Loeb, M.: Populations at risk for severe or complicated influenza illness: systematic review and meta-analysis. BMJ 347, f5061 (2013). https://doi.org/10.1136/bmj.f5061

22. Dutch Hospital Data. https://www.dhd.nl/klanten/Paginas/home. aspx (2019). Accessed 18 Apr 2019

23. WHO: Classification of Diseases (ICD) - ICD-10 Online Versions. https://www.who.int/classifications/icd/icdonlineversions/ en/ (2018). Accessed 26 Jul 2018

24. Dutch-Healthcare-Authority: Overview of the Dutch Healthcare Authority. https://www.nza.nl/english (2018). Accessed 15 Jun 2018

25. National-Health.Care-Institute. https://english.zorginstituutnederla nd.nl/ (2018). Accessed 15 Jun 2018

26. Hakkaart-van Roijen, L., van der Linden, N., Boumans, C., Kanters, T., Tan, S.S.: Costing manual: Methodology of costing research and reference prices for economic evaluations in healthcare [in Dutch: Kostenhandleiding: Methodologie van kostenonderzoek en referentieprijzen voor economische evaluaties in de gezondheidzorg] (2015)

27. SWAB: Management of community-Acquired Pneumonia in Adults: 2016 Guideline Update from the Dutch Working Party on Antibiotic Policy (SWAB) and Dutch Association of Chest Physicians (NVALT). https://www.swab.nl/swab/cms3.nsf/uploa ds/6A6E127F9A2C1168C125816F004A013A/\$FILE/CAP SWAB_2017-DEF_R5.pdf (2017). Accessed 19 Jul 2018

28. Hubben, G., Bootsma, M., Luteijn, M., Glynn, D., Bishai, D., Bonten, M., Postma, M.: Modelling the costs and effects of selective and universal hospital admission screening for methicillinresistant Staphylococcus aureus. PLoS ONE 6(3), e14783 (2011). https://doi.org/10.1371/journal.pone.0014783

29. Wassenberg, M.W., Kluytmans, J.A., Box, A.T., Bosboom, R.W., Buiting, A.G., van Elzakker, E.P., Melchers, W.J., van Rijen, M.M., Thijsen, S.F., Troelstra, A., Vandenbroucke-Grauls, C.M., Visser, C.E., Voss, A., Wolffs, P.F., Wulf, M.W., van Zwet, A.A., de Wit, G.A., Bonten, M.J.: Rapid screening of methicillin-resistant Staphylococcus aureus using PCR and chromogenic agar: a prospective study to evaluate costs and effects. Clin. Microbiol. Infect. 16(12), 1754-1761 (2010). https://doi.org/10.111 $1 /$ j.1469-0691.2010.03210.x

30. Statistics.Netherlands: Dutch Consumer Price index. https:// statline.cbs.nl/Statweb/publication/?DM $=$ SLNL\&PA $=71905$ ned $\& D 1=a \& D 2=0,95-117 \& H D R=T \& S T B=G 1 \& V W=T(2018)$. Accessed 8 Feb 2018

31. Gefenaite, G., Pistol, A., Popescu, R., Popovici, O., Ciurea, D., Dolk, C., Jit, M., Gross, D.: Estimating burden of 
influenza-associated influenza-like illness and severe acute respiratory infection at public healthcare facilities in Romania during the 2011/12-2015/16 influenza seasons. Influenza Other Respir. Viruses 12(1), 183-192 (2018). https://doi.org/10.1111/irv.12525

32. ECDC: Seasonal Influenza-Annual Epidemiological Report for 2015-16 Season. https://ecdc.europa.eu/sites/portal/files/docum ents/AER_for_2015-influenza-seasonal_0.pdf (2017). Accessed 5 Jul 2018

33. CDC: Laboratory-Confirmed Influenza Hospitalizations. https ://gis.cdc.gov/GRASP/Fluview/FluHospRates.html (2018). Accessed 5 Jul 2018

34. Snijders, B., van der Hoek, W., Stirbu, I., van der Sande, M.A., van Gageldonk-Lafeber, A.B.: General practitioners' contribution to the management of community-acquired pneumonia in The Netherlands: a retrospective analysis of primary care, hospital, and national mortality databases with individual data linkage. Primary Care Respir. J. 22(4), 400-405 (2013). https://doi.org/10.4104/ pcrj.2013.00085

35. Jain, S., Self, W.H., Wunderink, R.G., Fakhran, S., Balk, R., Bramley, A.M., Reed, C., Grijalva, C.G., Anderson, E.J., Courtney, D.M., Chappell, J.D., Qi, C., Hart, E.M., Carroll, F., Trabue, C., Donnelly, H.K., Williams, D.J., Zhu, Y., Arnold, S.R., Ampofo, K., Waterer, G.W., Levine, M., Lindstrom, S., Winchell, J.M., Katz, J.M., Erdman, D., Schneider, E., Hicks, L.A., McCullers, J.A., Pavia, A.T., Edwards, K.M., Finelli, L.: Communityacquired pneumonia requiring hospitalization among US adults. N. Engl. J. Med. 373(5), 415-427 (2015). https://doi.org/10.1056/ NEJMoa1500245

36. Marcos, P.J., Restrepo, M.I., Anzueto, A.: Community-acquired pneumonia requiring hospitalization. N. Engl. J. Med. 373(24), 2380-2381 (2015). https://doi.org/10.1056/NEJMc1511751

37. WHO: Estimates of Unit Costs for Patient Services for Spain. https ://www.who.int/choice/country/esp/cost/en/ (2018). Accessed 13 Sep 2018

38. WHO: Estimates of Unit Costs for Patient Services for Canada. https://www.who.int/choice/country/can/cost/en/ (2018). Accessed 8 Oct 2018

39. WHO: Estimates of Unit Costs for Patient Services for Italy. https ://www.who.int/choice/country/ita/cost/en/ (2020). Accessed 2 Feb 2020

40. WHO: Estimates of Unit Costs for Patient Services for Germany. https://www.who.int/choice/country/deu/cost/en/ (2020). Accessed 3 Feb 2020

41. Torner, N., Navas, E., Soldevila, N., Toledo, D., Navarro, G., Morillo, A., Perez, M.J., Dominguez, A., Working Group of the Project PI: Costs associated with influenza-related hospitalization in the elderly. Hum. Vaccin. Immunother. 13(2), 412-416 (2017). https://doi.org/10.1080/21645515.2017.1264829

42. Trucchi, C., Paganino, C., Orsi, A., Amicizia, D., Tisa, V., Piazza, M.F., Gallo, D., Simonetti, S., Buonopane, B., Icardi, G., Ansaldi, F.: Hospital and economic burden of influenza-like illness and lower respiratory tract infection in adults $\% 3 \mathrm{e} /=50$ years-old. BMC Health Serv. Res. 19(1), 585 (2019). https://doi. org/10.1186/s12913-019-4412-7

43. Haas, J., Braun, S., Wutzler, P.: Burden of influenza in Germany: a retrospective claims database analysis for the influenza season 2012/2013. Eur. J. Health. Econ. 17(6), 669-679 (2016). https:// doi.org/10.1007/s10198-015-0708-7

44. Ng, C., Ye, L., Noorduyn, S.G., Hux, M., Thommes, E., Goeree, R., Ambrose, A., Andrew, M.K., Hatchette, T., Boivin, G., Bowie, W., ElSherif, M., Green, K., Johnstone, J., Katz, K., Leblanc, J., Loeb, M., MacKinnon-Cameron, D., McCarthy, A., McElhaney, J., McGeer, A., Poirier, A., Powis, J., Richardson, D., Sharma, R., Semret, M., Smith, S., Smyth, D., Stiver, G., Trottier, S., Valiquette, L., Webster, D., McNeil, S.A., Serious Outcomes Surveillance Network of the Canadian Immunization Research Network I, Toronto Invasive Bacterial Diseases Network I: Resource utilization and cost of influenza requiring hospitalization in Canadian adults: a study from the serious outcomes surveillance network of the Canadian Immunization Research Network. Influenza Other Respir. Viruses 12(2), 232-240 (2018). https://doi.org/10.1111/ irv. 12521

45. van Werkhoven, C.H., Postma, D.F., Mangen, M.J., Oosterheert, J.J., Bonten, M.J., Group C.-S.s.: Cost-effectiveness of antibiotic treatment strategies for community-acquired pneumonia: results from a cluster randomized cross-over trial. BMC Infect. Dis. 17(1), 52 (2017). https://doi.org/10.1186/s12879-016-2179-6

46. Young-Xu, Y., van Aalst, R., Russo, E., Lee, J.K., Chit, A.: The annual burden of seasonal influenza in the US veterans affairs population. PLoS ONE 12(1), e0169344 (2017). https://doi. org/10.1371/journal.pone.0169344

47. Ortega-Sanchez, I.R., Molinari, N.A., Fairbrother, G., Szilagyi, P.G., Edwards, K.M., Griffin, M.R., Cassedy, A., Poehling, K.A., Bridges, C., Staat, M.A.: Indirect, out-of-pocket and medical costs from influenza-related illness in young children. Vaccine 30(28), 4175-4181 (2012). https://doi.org/10.1016/j.vaccine.2012.04.057

48. Federici, C., Cavazza, M., Costa, F., Jommi, C.: Health care costs of influenza-related episodes in high income countries: a systematic review. PLoS ONE 13(9), e0202787 (2018). https:// doi.org/10.1371/journal.pone.0202787

49. Statistics_Netherlands: Costs of Disease 2015. https://statline. rivm.nl/\#/RIVM/nl/dataset/50040NED/table?graphtype=Table \&ts $=1512975518824$. Accessed 12 Sep 2018

50. CDC: Information on Rapid Molecular Assays, RT-PCR, and other Molecular Assays for Diagnosis of Influenza Virus Infection. https://www.cdc.gov/flu/professionals/diagnosis/molecularassays.htm (2018). Accessed 10 Sep 2018

Publisher's Note Springer Nature remains neutral with regard to jurisdictional claims in published maps and institutional affiliations.

\section{Affiliations}

\section{Sierk D. Marbus ${ }^{1} \cdot$ Valentijn A. Schweitzer $^{2} \cdot$ Geert H. Groeneveld ${ }^{3}$. Jan J. Oosterheert ${ }^{4} \cdot$ Peter M. Schneeberger $^{5}$. Wim van der Hoek ${ }^{1}$. Jaap T. van Dissel ${ }^{1,3}$. Arianne B. van Gageldonk-Lafeber ${ }^{1}$. Marie-Josée Mangen ${ }^{1}$}

Valentijn A. Schweitzer

v.a.schweitzer-2@umcutrecht.nl

Geert H. Groeneveld

g.h.groeneveld@lumc.nl
Jan J. Oosterheert

j.j.oosterheert@umcutrecht.nl

Peter M. Schneeberger

p.schneeberger@jbz.nl 
Wim van der Hoek

wim.van.der.hoek@rivm.nl

Jaap T. van Dissel

jaap.van.dissel@rivm.nl

Arianne B. van Gageldonk-Lafeber

rianne.van.gageldonk@rivm.nl

Marie-Josée Mangen

marie-josee.mangen@rivm.nl

1 Centre for Infectious Diseases Epidemiology and Surveillance, Centre for Infectious Disease

Control (CIb), National Institute for Public
Health and the Environment (RIVM), PO Box 1,

3720 BA Bilthoven, The Netherlands

2 Julius Center for Health Sciences and Primary Care, University Medical Center Utrecht, Utrecht, The Netherlands

3 Department of Infectious Diseases and Internal Medicine, Leiden University Medical Center, Leiden, The Netherlands

4 Department of Internal Medicine and Infectious Diseases, University Medical Centre Utrecht, Utrecht, The Netherlands

5 Regional Laboratory for Medical Microbiology and Infection Prevention, 's-Hertogenbosch, The Netherlands 